\title{
Short-time dynamics of the positional order of the two-dimensional hard disk system
}

\author{
A. Jaster \\ Universität - GH Siegen, D-57068 Siegen, Germany
}

\begin{abstract}
We investigate the positional order of the two-dimensional hard disk model with short-time dynamics and equilibrium simulations. The melting density and the critical exponents $z$ and $\eta$ are determined. Our results rule out a phase transition as predicted by the Kosterlitz-Thouless-Halperin-Nelson-Young theory as well as a first-order transition.
\end{abstract}

Key words: Solid-liquid transition; Hard disk model; Short-time dynamics; Non-equilibrium kinetics PACS: 64.70.Dv, 05.70.Jk, 64.60.Fr, 64.60.Ht

Usually, numerical measurements of critical exponents are carried out from simulations in equilibrium using Monte Carlo (MC) techniques. Such simulations at or near the phase transition point, except for some special cases [1], are affected by the critical slowing down. Recently, Janssen, Schaub and Schmittmann [2] proposed an alternative, which allows their determination from the short-time dynamics. They discovered that a system with non-conserved order parameter (model A [3]) quenched from a high temperature state to the critical temperature shows universal short-time behaviour. This sets in after a microscopic time scale $t_{\text {mic }}$, during which the non-universal behaviour is swept away. Starting from a small value of the order parameter $m_{0}$ the order increases with a power law $M(t) \sim m_{0} t^{\theta}$, where $\theta$ is a new dynamic exponent. This short-time behaviour was supported by a number of MC simulations [4,5]. These investigations also provide a possibility to determine the conventional critical exponents. The exponents can be calculated from the time evolution of the second moment of the order parameter, the cumulant and additional observables, which evolve also with a power law. Since the simulations are performed in the early part of the dynamics, this method may eliminate critical slowing down.

While universal short-time behaviour was first seen when starting from un- 
ordered states, short-time dynamical scaling can also be found starting from the ordered state $(M(t=0)=1)$. In the latter case, no analytical calculations exist, but MC simulations have been performed [6,7,5]. Again, one can use the short-time behaviour to calculate the critical exponents, except for the new exponent $\theta$. In this letter, we use the dynamic relaxation of the twodimensional hard disk model starting from the ordered state to calculate the critical exponents for the positional order. Also this is the first time that the dynamic evolution in the early time is studied for a non-lattice model.

The nature of the two-dimensional melting transition is a long unsolved problem $[8,9]$. The Kosterlitz-Thouless-Halperin-Nelson-Young (KTHNY) theory [10] predicts two continuous transitions. The first transition occurs at temperature $T_{\mathrm{m}}$ when the solid (quasi-long-range positional order, long-range orientational order) undergoes a dislocation unbinding transition to the hexatic phase (short-range positional order, quasi-long-range orientational order). The second transition is the disclination unbinding transition (at $T_{\mathrm{i}}$ ) which transforms this hexatic phase into an isotropic phase (short-range positional and orientational order). There are several other theoretical approaches to the transition. An alternative scenario has been proposed by Chui [11]. He presented a theory via spontaneous generation of grain boundaries, i.e. collective excitations of dislocations, and predicted a conventional first-order phase transition from the solid to the isotropic phase. In this case, a region exists where both phases coexist instead of a hexatic phase. Even for the simple hard disk system no consensus about the nature of the transition has been established.

A number of simulations of the two-dimensional hard disk model in equilibrium have been performed. The melting transition of the hard disk system was first seen in a computer simulation by Alder and Wainwright [12]. They used a system of 870 disks and molecular dynamics methods (constant number of particles $N$, volume $V$ and energy $E$ simulations) and found that this system undergoes a first-order phase transition. But the results of such small systems are affected by large finite-size effects. Recent simulations used MC techniques either with constant volume ( $N V T$ ensemble) [13-16] or constant pressure ( $N p T$ ensemble) [17,18]. The analysis of Zollweg and Chester [13] for the pressure gave an upper limit for a first-order phase transition, but is compatible with all scenarios. Lee and Strandburg [17] used isobaric MC simulations and found a double-peaked structure in the volume distribution. Lee-Kosterlitz scaling led them to conclude that the phase transition is of first order. However, the data are not in the scaling region, since their largest system contained only 400 particles. MC investigations of the bound orientational order parameter via finite-size scaling with the block analysis technique of 16384 particle systems were done by Weber, Marx and Binder [14]. They also favoured a first-order phase transition. In contrast to this, Fernández, Alonso 
and Stankiewicz [18]】 predicted a one-stage continuous melting transition, i.e. a scenario with a single continuous transition. Their conclusions were based on the examination of the bond orientational order parameter in very long runs of different systems up to 15876 particles and hard-crystalline wall boundary conditions. Mitus, Weber and Marx [15] studied the local structure of a system with 4096 hard disks. From the linear behaviour of a local order parameter they derived bounds for a possible coexistence region. Finally, we showed by MC simulations in the $N V T$ ensemble [16] that a one-stage transition can be ruled out. The results of the bond orientational order parameter were compatible with the KTHNY predictions or a weak first-order transition.

In this letter, we present results for the positional order of the hard disk model obtained through MC simulations near the melting transition $\rho_{\mathrm{m}}$ and in the solid phase $\left(\rho>\rho_{\mathrm{m}}\right)$. First we use traditional simulations in equilibrium $(N V T$ ensemble) to locate the melting density $\rho_{\mathrm{m}}$ and to calculate the critical exponent $\eta$. After that, we investigate the dynamic relaxation at and above $\rho_{\mathrm{m}}$ (model $\mathrm{C}$ ) and calculate the critical exponents $z$ and $\eta$ from the short-time behaviour. We use always periodic boundary conditions and a rectangular box with ratio $\sqrt{3}: 2$, which is necessary since we start the relaxation from the ordered state. The disk diameter is set equal to one so that the lengths of the system are given by $L$ and $2 L / \sqrt{3}$, where $L=\sqrt{\sqrt{3} N / 2 \rho}$. For the simulations of the dynamic relaxation we use the conventional (local) Metropolis algorithm and choose the new positions of the particles (for symmetry reasons) with equal probability within a circle centered about its original position. Simulations in equilibrium are performed with a non-local Metropolis updating algorithm [20]. In this case, the new positions of the particles are chosen as usual within a square.

The $k$ th moment of the positional order parameter $\psi_{\text {pos }}$ is defined as

$$
\psi_{\text {pos }}^{k}=\left\langle\left|\frac{1}{N} \sum_{i=1}^{N} \exp \left(\mathrm{i} \overrightarrow{\mathrm{G}} \cdot \overrightarrow{\mathrm{r}}_{\mathrm{i}}\right)\right|^{k}\right\rangle
$$

where $\vec{G}$ is a reciprocal lattice vector and $\vec{r}_{i}$ denotes the position of particle $i$. The magnitude of $\vec{G}$ is given by $2 \pi / a$, where $a=\sqrt{2 / \sqrt{3} \rho}$ is the average lattice spacing. The orientation of $\vec{G}$ was defined in two different ways, which lead to two different $\psi_{\text {pos. }}$. In a first definition we fix the direction of $\vec{G}$ to that of a reciprocal lattice vector of the perfect crystal (which are unique due to the boundary condition of a rectangular box of ratio $2: \sqrt{3})$. The reason is that large crystal tilting (with the given boundary conditions) is not possible if we are in or near the solid phase, while small fluctuations of $\vec{G}$ are ignored.

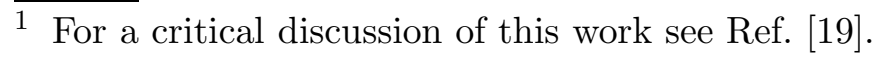


However, we use a second definition, where we determine the orientation of the crystal from the global bond orientation

$$
\psi_{6}=\frac{1}{N} \sum_{i=1}^{N} \frac{1}{N_{i}} \sum_{j=1}^{N_{i}} \exp \left(6 \mathrm{i} \theta_{\mathrm{ij}}\right) .
$$

The sum on $j$ is over the $N_{i}$ neighbours of the particle $i$ and $\theta_{i j}$ is the angle between the bond formed by particles $i$ and $j$ and an arbitrary but fixed reference axis. Neighbours are obtained by the Voronoi construction. Since we try to determine the global orientation of a single configuration, no average is taken in Eq. (2). With $\psi_{6} \sim \exp (6 \mathrm{i} \alpha)$, we define the angle $\alpha(0 \leq \alpha<\pi / 3)$ and therefore the orientation of the crystal and $\vec{G}$. Of course, the definition of $\vec{G}$ is only valid in a strict manner if the bond orientational correlation length $\xi_{6}$ is infinite (i.e. if we are in the hexatic or solid phase). Simulations show that this is probably the case [16]. However, if $\xi_{6}$ is finite, we have at least the case that $\xi_{6} \gg L$, since we simulate near the melting transition. Thus we have also in this case some kind of global orientation 2 . The simulations showed that the difference between the two definitions is negligible in the solid phase, while it is getting important below the transition point.

The positional correlation is quasi-long-ranged in the whole solid phase. Therefore, the fourth-order cumulant

$$
U=1-\frac{\psi_{\text {pos }}^{4}}{3\left(\psi_{\text {pos }}{ }^{2}\right)^{2}}
$$

(measured in the equilibrium) is independent of the size of the system for $\rho \geq \rho_{\mathrm{m}}$. We use this finite-size scaling (FSS) behaviour to locate the melting density $\rho_{\mathrm{m}}$ with simulations in equilibrium. Our results for the dependence of $U$ on $L$ for $N=32^{2}, 64^{2}$ and $128^{2}$ are shown in Fig. 1. We use the second definition of $\psi_{\text {pos }}$ (varying orientation of $\vec{G}$ ), since most of the simulations are performed below $\rho_{\mathrm{m}}$. However, the other definition leads to comparable results. The figure shows that the scale invariance of $U$ yields a melting density of $\rho_{\mathrm{m}} \approx 0.933$ (in reduced units). Since there is a tendency of the slope to decrease with larger systems, scale invariance may actually take place at a slightly larger density. However, even the value $\rho_{\mathrm{m}}=0.933$ is larger than the upper limit of a possible liquid-solid tie line of $\rho_{\mathrm{s}}=0.912$ [15] and $\rho_{\mathrm{s}}=0.904$ [13], respectively. The first result [15] was obtained by examining the behaviour of a local bond orientational order parameter as a function of the density. The second limit [13] was determined from a study of the pressure as a function of the density. Both simulations do not rule out a (one-stage or two-stage)

$\overline{2}$ Recent results concerning the liquid-solid structure of two-dimensional liquids can be found in Ref. [21]. 


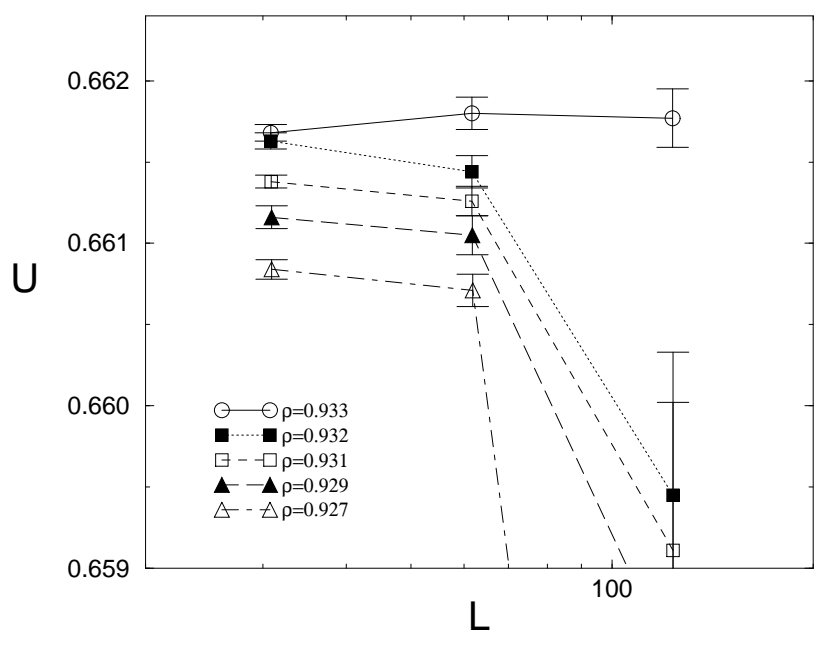

Fig. 1. FSS of the cumulant in the vicinity of $\rho_{\mathrm{m}}$.

continuous transition, but give limits for a conventional first-order transition from the liquid to the solid phase. However, for such a first-order scenario the melting density $\rho_{\mathrm{m}}$ (as determined from FSS of the positional order) must lie in the coexistence phase, i.e. below $\rho_{\mathrm{s}}$. Therefore, we can rule out a single first-order transition.

From the scaling relation $\psi_{\text {pos }}^{2} \sim L^{-\eta}$ we extract the critical exponent $\eta$ by a fit of all three system sizes. At $\rho=0.933$ we get $\eta=0.200(2)$, where the three points lie within statistical errors on a straight line. This result is incompatible with the prediction of the KTHNY theory of $1 / 4 \leq \eta\left(\rho_{\mathrm{m}}\right) \leq 1 / 3$ [10]. Thus we can rule out the KTHNY scenario, because of the measured exponent $\eta$. If our $\rho_{\mathrm{m}}$ would be determined too low, this result remains unchanged. We performed also simulations in equilibrium at $\rho=1.0$ (solid phase) to verify the scale invariance of $U$ above $\rho_{\mathrm{m}}$. FSS yields $\eta=0.0791(6)$.

We now come to the dynamic relaxation of the hard disk system starting from the ordered state. Simulations of the short-time dynamics of systems with quasi-long-range order were performed for the 6-state clock model [22], the XY model $[23,24]$ and the fully frustrated XY model $[25,24]$. From the scaling form of the second moment of the order parameter at or above $\rho_{\mathrm{m}}$

$$
\psi_{\operatorname{pos}}^{2}(t, L)=b^{-\eta} \psi_{\operatorname{pos}}^{2}\left(b^{-z} t, b^{-1} L\right)
$$

one obtains that the time dependence for sufficient large $L$ is given by a power law of the form [5]

$$
\psi_{\operatorname{pos}}^{2}(t) \sim t^{-\eta / z}
$$


Accordingly, FSS analysis of the time-dependent cumulant

$$
\tilde{U}(t)=\frac{\psi_{\text {pos }}^{4}(t)}{\left(\psi_{\text {pos }}^{2}(t)\right)^{2}}-1
$$

leads to

$$
\tilde{U}(t) \sim t^{d / z} .
$$

Therefore, one can determine the dynamic critical exponent $z$ from $\tilde{U}(t)$ and then, with $z$ in hand, the static exponent $\eta$ from the behaviour of $\psi_{\text {pos }}^{2}(t)$. In principle, one can also try to determine $\rho_{\mathrm{m}}$ from the short-time dynamic, as it was done for a second order transition [26]. However, this determination is difficult in systems with a KT-like transition $[23,5]$.

In the following we perform MC simulations at $\rho_{\mathrm{m}}$ and in the solid phase ( $\rho=1.0)$ to investigate the time evolution of ${\psi_{\text {pos }}}^{2}$ and $\tilde{U}$. For short-time simulations we use the first definition of $\vec{G}$ (fixed direction), since large crystal tilting is not possible if we study only the early part of the evolution. However, we checked that both definitions lead to similar results. Starting from the ordered state $\left(\psi_{\text {pos }}=1\right)$, i.e. with a perfect crystal with lattice spacing $a$, we release the system to evolve with the MC dynamic according to the Metropolis algorithm. We use systems of $8^{2}, 16^{2}, 32^{2}, 64^{2}$ and $128^{2}$ hard disks and measure the observables up to $5000 \mathrm{MC}$ sweeps. The average is taken over $\mathcal{O}(5000)$ samples for $N=128^{2}$ and $\mathcal{O}(300000)$ samples for $N=8^{2}$. The critical exponents are determined by least squares fits with a power law ansatz in the time interval $t=\left(t_{\min }, t_{\max }\right)$. Statistical errors are calculated by dividing the data into different subsamples. Systematic errors are estimated by the results of different system sizes and different time intervals, i.e. we examined the dependency of the slope from the fitted interval $t=\left(t_{\min }, t_{\max }\right)$ and number of particles $N$. The quoted error is a sum of the statistical and systematic error.

In Fig. 2 (a) we plot the time evolution of $\psi_{\text {pos }}{ }^{2}$ at the melting density for different system sizes in a double logarithmic scale. The figure shows that the power law behaviour starts after a microscopic time scale $t_{\text {mic }}$ of approximately $80 \mathrm{MC}$ sweeps. For times up to 500 the difference between the systems with $64^{2}$ and $128^{2}$ hard disks is negligible. Therefore, we omitted the data of $N=128^{2}$ in the plot. Figure 2 (b) shows the time dependence of $\psi_{\text {pos }}{ }^{2}$ for $N=128^{2}$ at both densities. In the time interval shown, the slope is nearly independent of time and yields $\eta / z=0.0990(5)$ at $\rho=0.933$ and $\eta / z=0.0385(7)$ at $\rho=1.0$, respectively.

To determine $z$ independently, we also measure the time evolution of the 

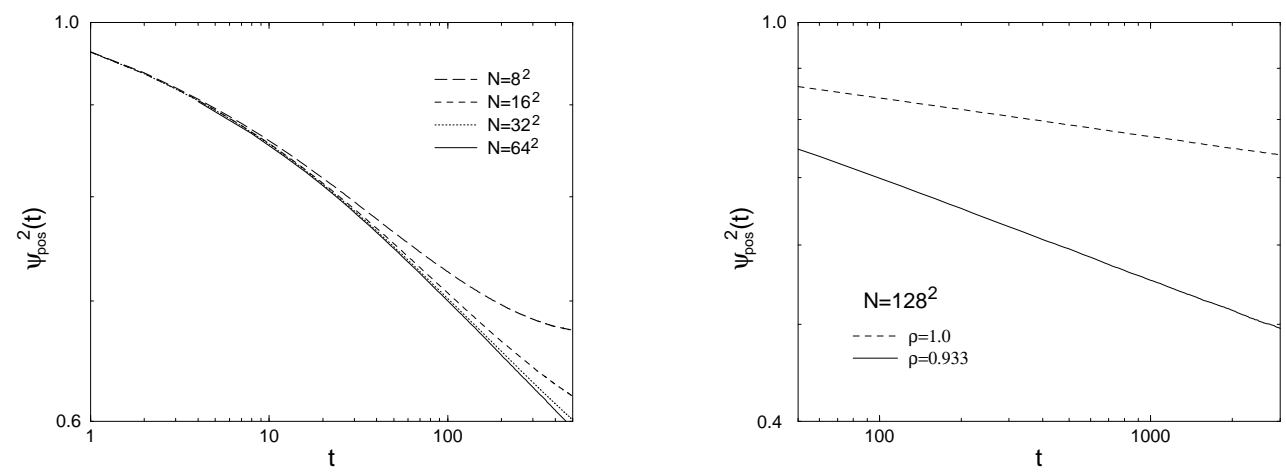

Fig. 2. (a) Time evolution of the second moment of the positional order parameter $\psi_{\text {pos }}^{2}$ starting from the ordered state at $\rho=0.933$. (b) $\psi_{\text {pos }}^{2}$ as a function of time for $\rho=0.933$ and $\rho=1.0$.

cumulant $\tilde{U}$. In Fig. 3 we show $\tilde{U}(t)$ at the melting density for two different system sizes. From the slope we get $z=2.01(2)$. The analysis for $\rho=1.0$ yields $z=2.06(4)$. Thus we get $\eta=0.199(3)$ at $\rho=0.933$ and $\eta=0.0794(29)$ at $\rho=1.0$. These values coincide with the results from FSS within statistical errors, as can be seen from Table 1 . Thus the data for $\eta$ are confirmed and new results for $z$ are produced.

In summary, we have performed short-time and equilibrium MC simulations of the hard disk model. The melting density $\rho_{\mathrm{m}}$ and the critical exponent $\eta$ are determined from simulations in equilibrium. The results rule out the KTHNY scenario as well as a single first-order transition from the solid to the liquid phase. The short-time behaviour was used to extract the critical exponents $\eta$ and $z$ at $\rho=\rho_{\mathrm{m}}$ and in the solid phase. The values of $\eta$ coincide with those

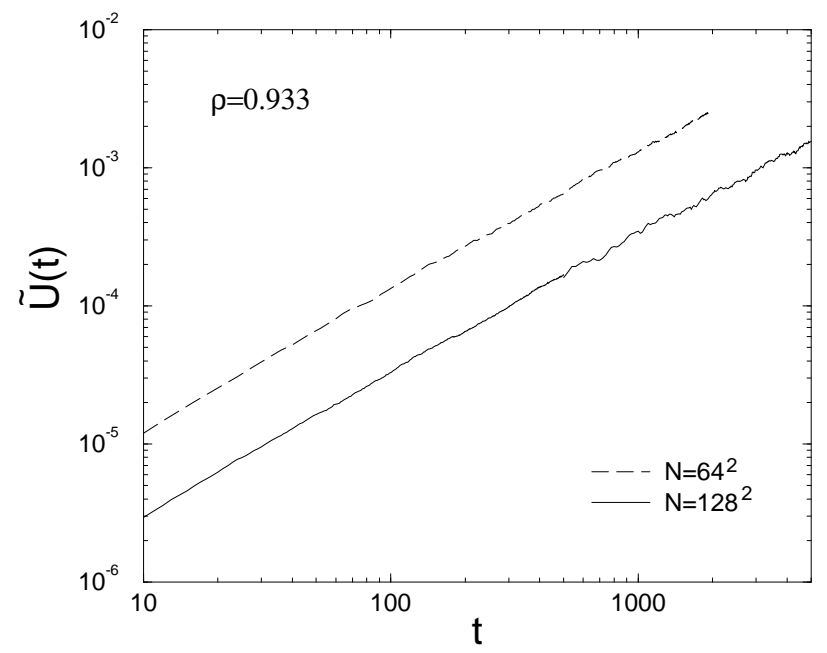

Fig. 3. Time dependent cumulant $\tilde{U}(t)$ at $\rho=0.933$ for $N=64^{2}$ and $N=128^{2}$. 


\begin{tabular}{cccc}
\hline \hline & \multicolumn{2}{c}{ short-time dynamics } & FSS \\
$\rho$ & $z$ & $\eta$ & $\eta$ \\
\hline 0.933 & $2.01(2)$ & $0.199(3)$ & $0.200(2)$ \\
1.0 & $2.06(4)$ & $0.0794(29)$ & $0.0791(6)$ \\
\hline \hline
\end{tabular}

Table 1

The critical exponents $z$ and $\eta$ determined from the short-time dynamics of the system and the value of $\eta$ measured with FSS methods.

from conventional FSS. Our simulations have shown that dynamic relaxation can also be used for this non-lattice model to measure the critical exponents.

Critical comments by Lothar Schülke are gratefully acknowledged. Especially we benefited from discussion with Elfie Prinz. This work was supported in part by the Deutsche Forschungsgemeinschaft under Grant No. DFG Schu 95/9-1.

\section{References}

[1] U. Wolff, Phys. Rev. Lett. 62 (1989) 361.

[2] H.K. Janssen, B. Schaub, B. Schmittmann, Z. Phys. B 73 (1989) 539.

[3] P.C. Hohenberg, B.I. Halperin, Rev. Mod. Phys. 49 (1977) 435.

[4] A.D. Huse, Phys. Rev. B 40 (1989) 304.

[5] A review of short-time dynamics is given in: B. Zheng, Int. J. Mod. Phys. B 12 (1998) 1419.

[6] D. Stauffer, Physica A 186 (1992) 197.

[7] Z.B. Li, L. Schülke, B. Zheng, Phys. Rev. E 53 (1996) 2940.

[8] K.J. Strandburg, Rev. Mod. Phys. 60 (1988) 161.

[9] M.A. Glaser, N.A. Clark, Adv. Chem. Phys. 83 (1993) 543.

[10] V.L. Berezinskii, Zh. Eksp. Teor. Fiz. 59 (1970) 907 [Sov. Phys. JETP 32 (1971) 493]; 61 (1971) 1144 [34 (1972) 610]; J.M. Kosterlitz, D.J. Thouless, J. Phys. C 6 (1973) 1181; J.M. Kosterlitz, J. Phys. C 7 (1974) 1046; B.I. Halperin, D.R. Nelson, Phys. Rev. Lett. 41 (1978) 121; A.P. Young, Phys. Rev. B 19 (1979) 1855 .

[11] S.T. Chui, Phys. Rev. Lett. 48, 933 (1982); Phys. Rev. B 28 (1983) 178.

[12] B.J. Alder, T.E. Wainwright, Phys. Rev. 127 (1962) 359.

[13] J.A. Zollweg, G.V. Chester, Phys. Rev. B 46 (1992) 11186. 
[14] H. Weber, D. Marx, Europhys. Lett. 27 (1994) 593; H. Weber, D. Marx, K. Binder, Phys. Rev. B 51 (1995) 14636.

[15] A.C. Mitus, H. Weber, D. Marx, Phys. Rev. E 55 (1997) 6855.

[16] A. Jaster, Europhys. Lett. 42 (1998) 277; Phys. Rev. E 59 (1999) 2594.

[17] J. Lee, K.J. Strandburg, Phys. Rev. B 46 (1992) 11190.

[18] J.F. Fernández, J.J. Alonso, J. Stankiewicz, Phys. Rev. Lett. 75 (1995) 3477; Phys. Rev. E 55 (1997) 750.

[19] H. Weber, D. Marx, Phys. Rev. Lett. 78 (1997) 398; J.F. Fernández, J.J. Alonso, J. Stankiewicz, Phys. Rev. Lett. 78 (1997) 399.

[20] A. Jaster, Physica A 264 (1999) 134.

[21] A.Z. Patashinski, A.C. Mitus, M.A. Ratner, Phys. Rept. 288 (1997) 409.

[22] P. Czerner, U. Ritschel, Phys. Rev. E 53 (1996) 3333.

[23] K. Okano, L. Schülke, K. Yamagishi, B. Zheng, J. Phys. A: Math. Gen. 30 (1997) 4527.

[24] H.J. Luo, B. Zheng, Mod. Phys. Lett. B 11 (1997) 615.

[25] H.J. Luo, L. Schülke, B. Zheng, Phys. Rev. E 57 (1998) 1327.

[26] L. Schülke, B. Zheng, Phys. Lett. A 215 (1996) 81. 